\title{
Machine Condition Monitoring Based on Transient Vibration Signal Analysis
}

\author{
Zhidong Chen ${ }^{\dagger}$ and C.K. Mechefske \\ Dept. of Mechanical and Materials Engineering, The University of Western Ontario, \\ London, Ontario, Canada N6G $5 B 9$
}

(Received 19 February 1999; accepted 21 June 2000)

\begin{abstract}
Most existing signal analysis methods are only suitable for stationary processes while transient vibration signals, due to their non-stationary nature, have been studied somewhat less. This paper seeks to find a method for the analysis of transient vibration signals and attempts to apply such a method to Machine Condition Monitoring. The Prony method is a modelling method that fits a linear combination of a series of exponential functions to sampled data. Because of its exponential base functions, this method is inherently suitable for analysing transient signals. The intention of this paper is to explore the application of the Prony method to the study of transient vibration signals and the potential of this method in Machine Condition Monitoring. This paper describes the preliminary work done to investigate this procedure as the basis of a useful machine condition monitoring and diagnostic technique. Also presented are preliminary numerical results of the Prony's model based frequency analysis of computer-generated, simulated vibration signals as well as experimentally-derived vibration signals.
\end{abstract}

${ }^{\dagger}$ Member of the International Institute of Acoustics and Vibration (IIAV)

\section{INTRODUCTION}

Over the past several decades a significant amount of research activity has been devoted to Machine Signature Analysis (MSA). ${ }^{1-3}$ The major tool used for MSA has been spectral analysis. Classical spectral estimation techniques include Periodogram, Averaged Periodogram and BlackmanTukey spectral estimation. Newer approaches to spectral analysis include a variety of Parametric modeling techniques. Within this category are the Rational Transfer Function Modelling Method, Autoregressive (AR) Power Spectral Density (PSD) Estimation, Moving Average (MA) PSD Estimation, Autoregressive Moving Average (ARMA) PSD Estimation, Prony Spectral Density Estimation and Maximum Likelihood Method (MLM)., ${ }^{2,3}$

The majority of the research and development work carried out to date with regard to signal processing strategies for machine condition monitoring and diagnostics applications has focused on signals generated from stationary processes. Non-stationary processes have been left relatively unstudied. Examples of methods that are applicable to non-stationary processes are the Wavelet and the Short Time Fast Fourier Transforms. ${ }^{1,4}$ The Prony method, originated by the French scientist Baron de Prony in $1795,{ }^{5}$ is also capable of analysing non-stationary processes ${ }^{5,6}$ and is inherently suitable for the study of exponentially decaying dynamic signals, such as those that develop as a result of many different types of machinery condition deterioration, ${ }^{6-9}$ for instance, induction motor current waveforms, ${ }^{6}$ power transformer tank vibrations ${ }^{7}$ and fluorescence decay behaviour for optical temperature sensing. ${ }^{8}$ This paper explores the use of the Prony method to study such transient signals.

The Prony method was originally used for interpolation between available data points. The method and its modified versions are techniques used for modelling data of equally spaced data samples. The Prony model is similar to the wellknown system identification techniques such as the AR and ARMA models. The Prony model seeks to fit an exponential model, which is a linear combination of a series of exponential functions, to sampled data. The Prony method first determines the linear prediction parameters that fit the sampled data. Such linear prediction parameters are then used as coefficients to form a polynomial. The roots of this polynomial are finally employed to estimate the damping coefficients, the sinusoidal frequencies, the exponential amplitude and the sinusoidal initial phase of each of the exponential terms.

\section{THE PRONY METHOD}

Assuming one has $N$ complex data samples $x[1], \ldots, x[N]$, the Prony method estimate $x[n]$ with a $p$-term complex exponential model is:

$$
\hat{x}[n]=\sum_{k=1}^{p} A_{k} \exp \left[\left(a_{k}+j 2 \pi f_{k}\right)(n-1) T+j \theta_{k}\right] ; \quad 1<n<N
$$

where $T$ is the sample interval, $A_{k}$ is the amplitude, $\alpha_{k}$ is the damping coefficient, $f_{k}$ is the frequency and $\theta_{k}$ is the initial phase.

Rewrite Eq. (1) as

$$
\hat{x}=\sum_{k=1}^{p} h_{k} Z_{k}^{n-1}
$$

where

$$
\begin{gathered}
h_{k}=A_{k} \exp \left(j \theta_{k}\right) ; \\
Z_{k}=\exp \left[\left(\alpha_{k}+j 2 \pi f_{k}\right) T\right] .
\end{gathered}
$$

